\title{
EchoGéo
}

\section{Commerce et logiques d'acteurs dans la région de Kolda au Sénégal}

Le cas du lait de vache

\section{Néné Dia}

\section{OpenEdition \\ Journals}

\section{Édition électronique}

URL : https://journals.openedition.org/echogeo/11048

DOI : 10.4000/echogeo. 11048

ISSN : 1963-1197

\section{Éditeur}

Pôle de recherche pour l'organisation et la diffusion de l'information géographique (CNRS UMR 8586)

\section{Référence électronique}

Néné Dia, «Commerce et logiques d'acteurs dans la région de Kolda au Sénégal », EchoGéo [En ligne], 8 | 2009, mis en ligne le 25 mars 2009, consulté le 31 juillet 2021. URL : http:// journals.openedition.org/echogeo/11048; DOI : https://doi.org/10.4000/echogeo.11048

Ce document a été généré automatiquement le 31 juillet 2021.

EchoGéo est mis à disposition selon les termes de la licence Creative Commons Attribution - Pas d'Utilisation Commerciale - Pas de Modification 4.0 International (CC BY-NC-ND) 


\section{Commerce et logiques d'acteurs dans la région de Kolda au Sénégal}

Le cas du lait de vache

Néné Dia

\section{Introduction}

Située au sud du Sénégal (cf. carte 1) et entourée de trois pays (Gambie, Guinée Bissau et Guinée Conakry), la région de Kolda ou Haute Casamance est la première région productrice de coton du pays, et la deuxième pour l'arachide. La Haute Casamance est également la deuxième région d'élevage du Sénégal ${ }^{1}$ tant en raison des effectifs bovins (19\% du cheptel national selon la Direction de la Prévision Statistique, 2004) que d'un mode de vie et d'une identité culturelle marqués par l'élevage. Contrairement à la première région d'élevage du pays, le Ferlo, où le commerce du cheptel vif domine, pratiqué par des éleveurs nomades, en Haute Casamance, les éleveurs sont sédentaires et le commerce du lait de vache constitue le pilier de l'économie pastorale depuis 1998. Avant cette date, le lait y était en grande partie autoconsommé par les producteurs, faute de débouchés. 


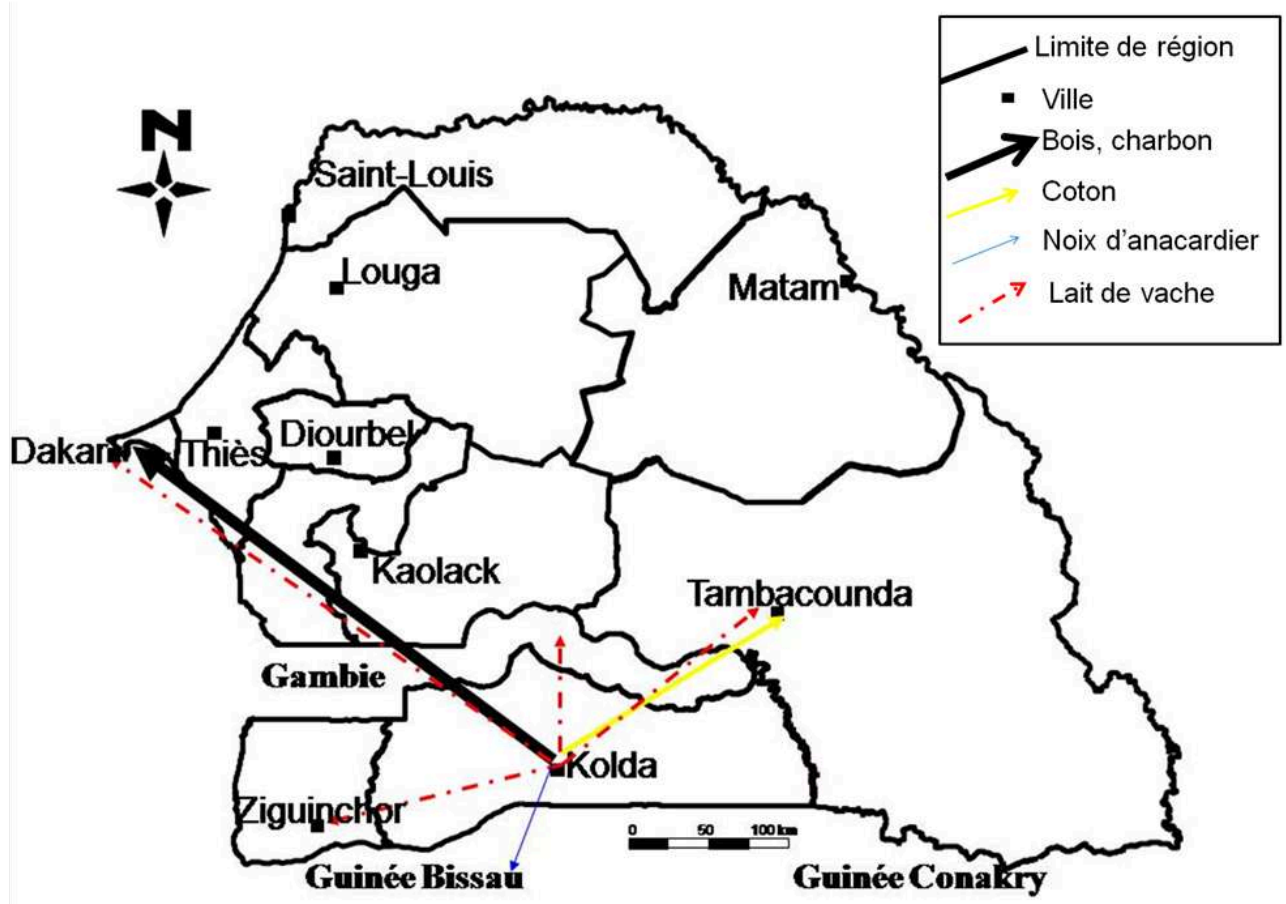

Grâce à la création des mini-laiteries (petites entreprises de transformation artisanale du lait) en 1998, le lait de vache est devenu source de revenus grâce à l'existence d'aires de collecte. Ces mini-laiteries sont approvisionnées par les agropasteurs qui résident dans les villages situés à la périphérie des deux principales villes de la région de Kolda : Kolda et Vélingara. Cet espace de collecte est nommé " ceinture laitière périurbaine " (Dièye et al, 2003). L'intervention de la principale société de production cotonnière et de développement rural du Sénégal, Société de Développement et des Fibres Textiles $(\text { SODEFITEX })^{2}$, favorise une extension du rayon de collecte de vache au-delà de l'espace périurbain de Kolda. La SODEFITEX intervient notamment à travers une de ses structures : la Base d'Appui aux Méthodes et Techniques pour l'Agriculture, les autres Activités Rurales et l'Environnement (BAMTAARE ${ }^{3}$ ). Il s'agissait pour la SODEFITEX de diversifier ses activités dans un contexte marqué par une baisse des cours mondiaux du coton.

Prestataire de services, la BAMTAARE met en place un « bassin laitier » à partir de 2006 en installant des centres de collecte frigorifiques appelés tanks (cf. croquis 1). Son objectif est la commercialisation du lait de vache dans des villes comme Ziguinchor, Kaolack, Thiès ou Dakar. Le « bassin laitier » a été constitué au moment où il y avait une inflation des prix du lait importé ${ }^{4}$ sous forme de poudre au Sénégal. Ce contexte était $a$ priori favorable au développement du commerce du lait de vache dans les grands centres urbains. Pourtant, les mini-laiteries et la BAMTAARE ne rencontrent pas le succès escompté. Les aléas liés à la production laitière (insuffisance du lait, mévente due à la concurrence de la poudre de lait importé) sont autant de contraintes qui minent la filière laitière en Haute Casamance. Les difficultés actuelles de cette filière révèlent, entre autres, la fragilité de certains acteurs disposant pourtant de moyens logistiques et financiers importants (BAMTAARE), mais également la solidité des petites entreprises de transformation artisanale du lait. 
Dans cet article, il s'agit donc d'étudier le fonctionnement et les limites des systèmes de collecte de lait de Haute Casamance. Pour comprendre ces systèmes de collecte, les stratégies d'approvisionnement des acteurs et la commercialisation du lait de vache, nous avons effectué des entretiens avec les propriétaires de mini-laiteries, les fournisseurs et les responsables de la BAMTAARE. Les résultats tirés de l'analyse des enquêtes de terrain ${ }^{5}$ dans la région de Kolda, complétés par l'étude de la bibliographie, sont organisés en deux points. Le premier aborde le processus de mise en place des ceintures périurbaines et $\mathrm{du}$ "bassin laitier ». Le second analyse les stratégies des acteurs (BAMTAARE et propriétaires des mini-laiteries) et leur fragilité face aux aléas de la production et de la commercialisation.

\section{Constitution des ceintures laitières périurbaines et du « bassin laitier»}

5 Dans les années 1960-1990, le lait de vache était principalement destiné à l'autoconsommation en Haute Casamance. Pendant cette période, la production de lait par les vaches trypanotolérantes (résistantes à la maladie du sommeil) s'arrêtait en saison sèche. En effet, ces vaches sont beaucoup plus connues pour leur aptitude à l'embouche bovine qu'à la production laitière ( $\mathrm{Ba}, 1986)$. Mais l'introduction d'une innovation technique en 1985 par la recherche agronomique a changé la donne: la stabulation permet d'avoir une production laitière continue en saison sèche grâce à l'utilisation de compléments alimentaires comme la graine de coton (Fall, Faye, 1992). Initiés par le Centre de Recherches Zootechniques de Kolda, les agro-pasteurs résidant dans les villages situés à la périphérie des villes de Kolda et de Vélingara utilisent désormais cette technique. Un espace de collecte se mit progressivement autour de Kolda en 1998 ou de Vélingara en 2000, avec la création des unités de transformation artisanale (Dièye et al, 2003). Les aires de collecte des mini-laiteries constituent les ceintures laitières périurbaines respectives de Kolda et de Vélingara.

6 Ces mini-laiteries pasteurisent le lait avant de le commercialiser en Haute Casamance. Si la ville de Kolda compte 6 mini-laiteries contre seulement 2 à Vélingara, c'est en raison des débouchés, plus intéressants dans le premier cas. En effet, Kolda a une population de 60000 habitants, la ville de Vélingara le tiers seulement. En outre, chaque mini-laiterie de Kolda collecte en moyenne 300 litres de lait par jour, pour 200 litres quotidien à Vélingara.

7 Cependant, à partir de 2004, on note la présence de mini-laiteries non plus seulement dans les villes de Kolda et de Vélingara, comme c'était le cas avec les premières unités, mais aussi en milieu rural. Il s'agit par exemple de l'unité de transformation artisanale de lait apparue dans le village de Kounkané en 2004 (département de Vélingara) et de celle de Bantancountou Maoundé, installée en 2007 (département de Kolda). Les minilaiteries rurales sont approvisionnées par les producteurs résidant dans un rayon de $15 \mathrm{~km}$. Contrairement à la ceinture périurbaine, où la nature des échanges est ruralurbain, ici ce sont les relations de type rural-rural qui prédominent aussi bien dans la collecte que dans la commercialisation. La distance par rapport à la ville et les surcoûts liés au transport expliquent le choix des propriétaires des mini-laiteries rurales de se limiter à l'espace rural. De plus, le nombre d'habitants dans les villages de Kounkané et Bantancountou Maoundé (200 et 1000 habitants) permet aux mini-laiteries rurales d'y 
écouler le tiers de leur production journalière (100 à 150 litres). Le reste est vendu dans les villages environnants et lors des marchés hebdomadaires.

Le rayon de collecte des mini-laiteries urbaines ne dépasse cependant pas $35 \mathrm{~km}$ du fait de l'absence d'infrastructures de conservation dans les villages d'agropasteurs (Dièye, 2006). Les éleveurs transportent eux-mêmes le lait aux mini-laiteries, dans des bidons de 20 litres. Or, au-delà de $35 \mathrm{~km}$, le temps du transport induit un risque de caillage du lait, ce qui le rend impropre à la pasteurisation. Disposant de capitaux importants, la BAMTAARE surmonte ces handicaps liés au transport du lait.

En dehors des actions classiques de vulgarisation de la culture cotonnière, la SODEFITEX participe à la valorisation du lait de vache en Haute Casamance à travers sa structure d'appui au développement rural. Il s'agit de la Direction du Développement Rural (DDR) créée en 1986 et appelée la BAMTAAREdepuis 2003. Prestataire de services pour les structures étatiques ou privées, la BAMTAARE encadre les producteurs du sud du Sénégal dans l'optique des programmes étatiques de diversification de l'agriculture sénégalaise (cultures de maïs, de sésame de manioc ou de tournesol pour le projet biocarburant). La BAMTAARE a également géré le projet étatique lancé en 2000 pour le financement des petites entreprises de transformation artisanale de lait (Projet des Petites et Grandes Laiteries). Après la fin de ce projet (2004), la BAMTAARE a mis en place son propre programme d'exploitation du lait de vache, appelé «bassin laitier ", en 2006. L'objectif est de collecter le lait de vache au-delà des ceintures laitières périurbaines.

Croquis 1

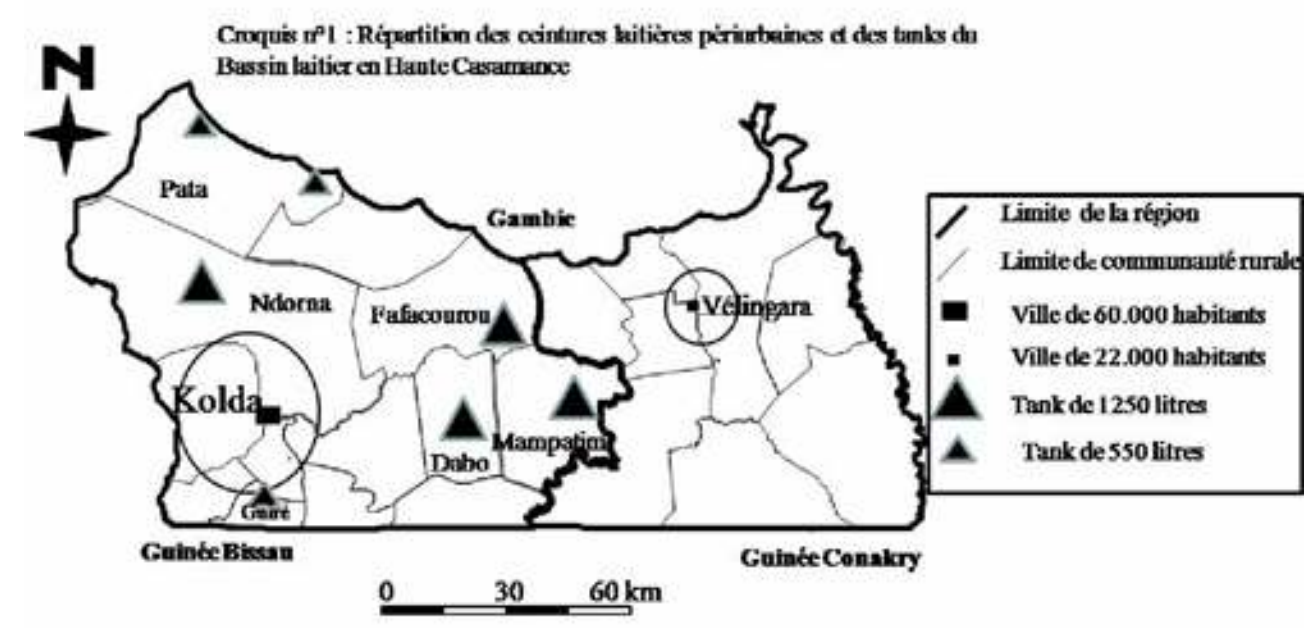

Sonnce : Nanè DSa

La BAMTAARE a installé 7 centres de refroidissement du lait trait ou tanks (cf. croquis 1). Ils constituent d'abord des points de collecte de lait, polarisant plusieurs villages dans un rayon moyen de $20 \mathrm{~km}$. L'emplacement du tank dans un village dépend de la localisation géographique de ce dernier par rapport aux voies de communication. En effet, il doit être desservi par les routes ou les pistes. Le tank le plus proche de la ceinture laitière périurbaine de Kolda se trouve à $20 \mathrm{~km}$ au sud de la ville de Kolda et le plus éloigné est à Ngoudourou, à $100 \mathrm{~km}$ au nord (cf. croquis 1). Tous les 3 jours, un 
camion de ramassage récupère le lait stocké dans les centres de collecte pour le transporter jusqu'à la ville Kolda. Ce fonctionnement, adopté par la BAMTAARE, s'adapte bien à l'espace de production en raison de la dispersion géographique des producteurs et du nombre important de leurs villages (environ 150). Ainsi, cette méthode semble être une bonne option pour l'organisation spatiale d'un système de collecte à grande échelle. Le lait collecté est finalement vendu par la BAMTAARE dans les villes situées à l'ouest du Sénégal.

11 Le transport du lait du «bassin laitier» jusqu'aux lieux de vente suppose l'existence d'un camion frigorifique pour éviter la détérioration du produit. Or, ce transport se paie en fonction du kilométrage. On retrouve ce même système dans d'autres pays comme le Tchad, où le transport du lait est effectué par des "quarantiers", transporteurs rémunérés au kilomètre (Duteurtre, 2005). La BAMTAARE joue le même rôle que les "quarantiers", car le lait est collecté dans des zones relativement enclavées, à plus de $50 \mathrm{~km}$ de distance de la ville de Kolda, avant d'être commercialisé dans certaines villes du Sénégal. Il s'agit de centres urbains proches comme Tambacounda, Ziguinchor ou plus éloignés comme Kaolack, Thiès ou la capitale sénégalaise Dakar. La population de Kaolack et Thiès est de 100000 à 200000 habitants et celle de Dakar 1500000 habitants; ces espaces urbains offrent donc de meilleures opportunités de commercialisation. Contrairement aux mini-laiteries qui ne disposent pas des moyens logistiques pour le transport du lait, la BAMTAARE livre du lait non transformé. Les ventes s'effectuent sous forme de commandes de 400 litres pour les centres urbains distants de moins de $100 \mathrm{~km}$ de la ville de Kolda (Tambacounda, Ziguinchor) ou de 3.000 litres pour ceux qui sont au-delà de $100 \mathrm{~km}$ (Kaolack, Thiès ou Dakar). La BAMTAARE a donc permis l'extension à la fois des aires de collecte et de commercialisation en dehors de la région de Kolda.

12 Ainsi, la ressource que représente le lait de vache est mise en valeur grâce aux réseaux tissés par les acteurs de niveau local depuis 1998 (producteurs, propriétaires des minilaiteries) mais aussi grâce aux acteurs de dimension industrielle (la BAMTAARE), depuis 2006. Par conséquent, ces acteurs mettent en place différentes stratégies pour la collecte et la commercialisation; celles mises en place par les mini-laiteries et la BAMTAARE sont révélatrices de la fragilité de leur système de collecte.

\section{Les stratégies des acteurs du commerce du lait de vache}

13 Malgré la pratique de la stabulation laitière, le principal problème reste l'insuffisance de la production laitière en saison sèche. A cette période, la demande en lait est supérieure à l'offre en Haute Casamance. Pour sécuriser leurs approvisionnements en lait en fidélisant les éleveurs, diverses tactiques sont mises en place par la BAMTAARE et les propriétaires des mini-laiteries. Des relations personnalisées s'établissent entre fournisseurs et acheteurs (Alvarez, 1997) et les mini-laiteries sont les premières à exploiter ce créneau des relations non marchandes.

14 Le cas de la mini-laiterie urbaine de Vélingara, Larogal, enconstitue un exemple. Larogal est le seul Groupement d'Intérêt Economique (GIE) d'agropasteurs qui possède sa minilaiterie dans la région de Kolda. Dans ce cas particulier, c'est une organisation non gouvernementale française (Agronomes et Vétérinaires Sans Frontières) qui a avancé les fonds pour le fonctionnement de la mini-laiterie. Le système repose sur une 
sélection des membres avant l'entrée dans le GIE. Tout d'abord, l'agropasteur doit être capable de fournir 80 litres de lait par mois, avoir une étable pour la stabulation laitière et payer une cotisation annuelle de 2500 FCFA. La confiance mutuelle et une forte cohésion sociale sont fondamentales dans cette organisation. En 2007, étant donné que cette mini-laiterie fonctionnait correctement, aucun élargissement au-delà des 32 éleveurs adhérents depuis 2004 n'était envisagé, malgré les nombreuses demandes d'entrées dans le GIE (Magrin, 2007). L'étroitesse du marché de Vélingara (22 000 habitants) pousse également les gérants à ne pas augmenter la production. L'objectif est d'éviter les invendus, d'autant qu'il existe des mini-laiteries concurrentes. Cette organisation présente un atout indéniable : les producteurs sont fidèles et fournissent régulièrement du lait parce qu'il s'agit de leur entreprise. Ils sont impliqués, et informés des charges et des dépenses liées à la transformation laitière. Cependant, ce modèle est difficilement reproductible en dehors de situations où il existe une forte cohésion sociale. Le système Larogal constitue un cas exceptionnel dans le paysage des mini-laiteries de la région de Kolda.

Bien que le nombre de membres soit limité, la mini-laiterie est également fournie par des non adhérents comme les éleveurs transhumants originaires de la Gambie. Les pasteurs gambiens, les soninkés, sont sollicités quand l'approvisionnement en lait fait défaut comme cela a été le cas en 2007. Cette stratégie est également utilisée par les propriétaires des autres unités de transformation de lait de la ville de Vélingara et du village de Kounkané. Tous ces moyens mis en œuvre visent à garantir l'approvisionnement en lait durant la saison sèche. Hormis ces stratégies de sécurisation de la matière première - le lait de vache -, l'aspect relationnel est aussi déterminant pour les propriétaires des mini-laiteries rurales et urbaines.

En s'installant dans leur milieu d'origine, les propriétaires des mini-laiteries rurales de Kounkané et Bantancountou Maoundé s'appuient sur un puissant réseau familial. Les gérants de ces mini-laiteries rurales ont pu ainsi se constituer une clientèle fidèle auprès des agropasteurs. Pour comprendre le choix d'un éleveur, il faut prendre en compte les solidarités familiales (Vatin, 1996). Les relations non marchandes jouent un rôle majeur dans ces localités où le sentiment communautaire est fort. Cet aspect a été souligné par des auteurs évoquant le capital social ou des raisons non marchandes (Loudiyi et al, 2006 ; Vatin, 1996). Les propriétaires des mini-laiteries rurales n'ont pas été les seuls à exploiter le filon social. Leurs homologues de la ville de Kolda n'hésitent pas à mettre en place des tactiques pour fidéliser les producteurs de lait de la ceinture périurbaine, qui sont très sollicités.

Les propriétaires des mini-laiteries urbaines de Kolda utilisent des stratégies différentes, comme la fourniture de services. La cession de graines de coton à crédit est par exemple utilisée comme moyen de fidélisation efficace. Ne disposant pas des moyens financiers suffisants pour passer une commande auprès de la SODEFITEX, les fournisseurs de lait s'approvisionnent auprès des mini-laiteries. Le prix de la graine de coton est ensuite soustrait des paiements mensuels ou bi-mensuels versés aux producteurs de lait. Certaines mini-laiteries vont encore plus loin en mettant une boutique à la disposition des producteurs. Ceux qui en ont besoin achètent à crédit des denrées alimentaires, remboursées à la fin du mois par le même système. Ce fonctionnement perdure même lors de certaines grandes occasions comme les fêtes religieuses ou la rentrée des classes. Ces stratégies de fidélisation tentent de réduire 
l'incertitude à laquelle sont confrontés les acteurs de la filière laitière dans le domaine de la production (Dièye, 2006). Pour autant, elles ne constituent pas une garantie.

L'installation d'un tank de 550 litres par la BAMTAARE dans un village situé au sud de la ceinture périurbaine (cf. croquis 1 ) de Kolda est à l'origine d'un emboîtement entre les aires de collecte des ceintures périurbaines et du «bassin laitier ». Les producteurs de lait préfèrent désormais livrer directement au tank plutôt que de parcourir, à vélo, les $20 \mathrm{~km}$ nécessaires pour vendre leur lait aux mini-laiteries de Kolda. La principale conséquence est l'arrêt de l'approvisionnement en lait, au détriment des mini-laiteries urbaines. Malgré tout, les mini-laiteries disposent toujours de la quantité de lait suffisante pour fonctionner après la saison des pluies. Grâce à leur expérience dans la production laitière, leurs propriétaires s'adaptent mieux aux aléas liés à l'approvisionnement que les centres de collecte de la BAMTAARE.

La BAMTAARE a intégré de nombreux villages dans son aire de collecte et a fourni gratuitement des graines de coton pour sécuriser l'approvisionnement en lait. Pourtant, la production laitière reste très insuffisante. A l'exception des fournisseurs du tank de lait inclus dans la ceinture périurbaine de Kolda (cf. croquis 1), ceux des autres centres du "bassin laitier» ne maitrisent pas la stabulation. D'après nos enquêtes, seuls $36 \%$ des producteurs de lait pratiquent la stabulation. Pour permettre au veau de survivre en saison sèche, $64 \%$ des producteurs de lait suspendent la livraison à destination des tanks. En effet, dans les systèmes d'élevages africains, on recherche beaucoup plus la sécurité que la rentabilité (Faye, 2006). Or, cette dimension n'a pas été prise en compte par les concepteurs du «bassin laitier ", de même que le problème de la cohabitation entre agriculture et élevage. Le tank installé dans la communauté rurale de Pata (croquis 1) en est révélateur; Pata dispose d'une forêt classée qui est le lieu privilégié d'extension de la culture arachidière. Certains auteurs (Fanchette, 2002) ont souligné les conflits existant entre agriculteurs et éleveurs, ce qui explique les difficultés d'approvisionnement du tank en saison des pluies, période où les troupeaux sont confiés à des bergers qui migrent hors de ces zones de fortes cultures ; c'est la période de la petite transhumance effectuée par les agro-pasteurs (Ba, 1989 : 11). A ces problèmes de collecte s'ajoutent ceux de la commercialisation du lait.

Commercialisant sa production dans ce circuit interrégional, la BAMTAARE est plus exposée que les mini-laiteries à la concurrence avec la poudre de lait importé. La plupart des industries de transformation laitière utilise la poudre de lait, moins chère que le lait produit dans le «bassin laitier ». En raison des surcoûts liés à son transport, le lait de vache est finalement commercialisé dans les villes plus proches telles Tambacounda ou Ziguinchor. Cependant, ce marché n'absorbe pas la totalité de l'offre du «bassin laitier» qui est de 3000 litres en moyenne par semaine. La faible disponibilité du lait en saison sèche et sa mévente généralisée ont ainsi expliqué la suspension des activités de collecte de février 2007 à juin 2008 dans le « bassin laitier ».

21 Ne disposant pas des mêmes moyens logistiques que la BAMTAARE, les minilaiteries écoulent le lait de vache transformé dans les villes de Kolda, de Vélingara et sur les marchés hebdomadaires de Haute Casamance. L'autre raison de ce confinement du commerce laitier dans la ville de Kolda est l'insuffisance de la production laitière locale. Elle a surtout été provoquée, selon les principaux acteurs de la filière, par la rareté de la graine de coton en 2007. Principal complément alimentaire des vaches laitières, cette graine est appréciée des éleveurs sénégalais, notamment parce que cet aliment de bétail est plus accessible que ceux fabriqués industriellement, comme les 
tourteaux d'arachide. La forte demande en 2007 a été à l'origine d'une pénurie en graine de coton, cause d'une baisse significative de la production laitière. Ce déficit a poussé les propriétaires des mini-laiteries à écouler le lait dans les villes de Kolda et de Vélingara.Le commerce du lait demeure une activité qui participe à la différentiation de ses terroirs.

\section{Photos - Les différences entre collecte et commercialisation}
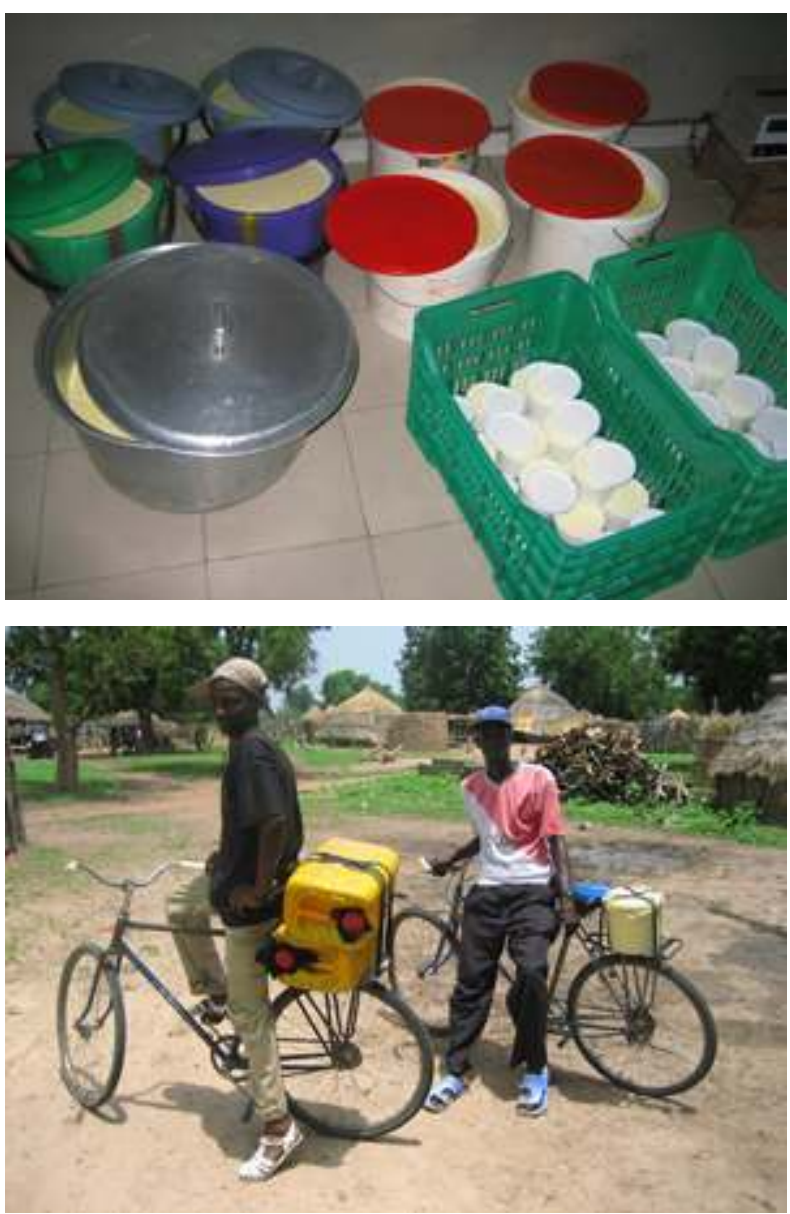

Collecté journellement sur une distance de 15 à $30 \mathrm{~km}$ des villes de la région de Kolda, le lait de vache pasteurisé et vendu par les 10 mini-laiteries à la fois dans les villes de Kolda, de Vélingara et sur les marchés hebdomadaires ruraux 


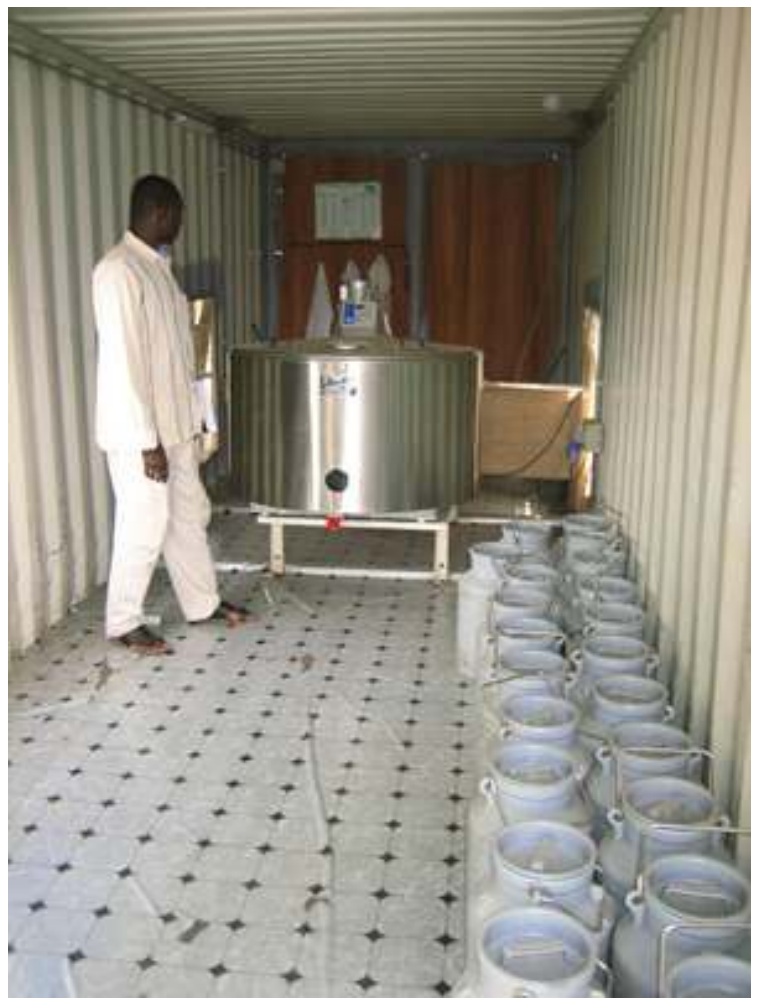

Par contre, dans le bassin laitier, des tans frigorifiques conservent le lait pendant 3 jours. Non transformé, ce lait est en grande partie commercialisé hors de la région de Kolda (ville de Ziguinchor, Tambacounda et Dakar).

Source : Les 3 clichés sont de Géraud Magrin.

Toutefois, en saison des pluies, il y a une surproduction de lait. Les marchés identifiés comme lieux d'écoulement potentiels (Dakar et Kaolack) bénéficient également d'un afflux d'éleveurs. De ce fait, l'offre est supérieure à la demande, ce qui n'est pas un phénomène nouveau ( $\mathrm{Ba}, 1986)$. Mais les mini-laiteries, et surtout la BAMTAARE, se trouvent dans l'incapacité de commercialiser leur lait dans le circuit interrégional durant cette période.

\section{Conclusion}

Produit essentiellement autoconsommé jusque dans les années 1990, le lait de vache a acquis en quelques années un statut de produit commercial dans la région, à partir de la constitution des ceintures périurbaines en 1998, puis du "bassin laitier» de la SODEFITEX en 2006. Sa collecte au sein de ces espaces suscite des concurrences entre les propriétaires des mini-laiteries et la BAMTAARE. Toutefois, ces acteurs n'ont pas la même capacité de réaction face aux incertitudes inhérentes à la production laitière locale. Bien que disposant de moyens financiers et logistiques supérieurs, la BAMTAARE bute sur les difficultés liées à la collecte et à la commercialisation du lait hors de la région de Kolda. En saison sèche, les activités de collecte du lait sont interrompues au sein $d u$ «bassin laitier» au moment où le marché est rentable. En revanche, l'expérience des propriétaires de mini-laiteries, les relations privilégiées qu'ils entretiennent avec leurs fournisseurs et la pratique de la stabulation expliquent la continuité des activités de collecte en saison sèche dans les ceintures périurbaines. 
Toutefois, la filière laitière reste fragile, car elle est soumise à des aléas comme l'insuffisance de la production laitière, les difficultés de commercialisation du lait hors de l'espace régional et la concurrence de la poudre de lait importée. Toutes ces incertitudes induisent son faible poids dans l'économie de Haute Casamance à côté des activités agricoles et forestières. Néanmoins, le commerce laitier périurbain peut avoir un pouvoir structurant sur l'espace urbain et renforcer la polarisation des villes de Kolda et Vélingara à travers les services de collecte, de transformation et de commercialisation.

\section{BIBLIOGRAPHIE}

Alvarez A., 1997. Marchés du lait et autosuffisance nationale : le rôle de la filière tropicale au Mexique, Cahiers des Sciences Humaines 30 (4) : 667-686.

Ba C., 1986. Les Peuls du Sénégal, étude géographique, Dakar, NEA, 394 p.

Boulet J., 2001. Systèmes de production agricole et gestion de l'espace au Sénégal oriental et en Haute Casamance, Dakar, Programme Sénégal Oriental, 149 p.

Broutin C., Duteurtre V., Tandia A., Touré B., François M., 2005. « Accroissement et diversification de l'offre de produits laitiers au Sénégal : la bataille industrielle du lait en poudre à Dakar et desmini-laiteries à la conquête des marchés des villes secondaires ", communication à l'atelier vers de nouvelles politiques laitières,Bamako 29 mai- 2juin.

Dieye P. N., Duteurtre G., Sissoko M. M., Sall M., Dia D., 2003. La mini laiterie et la valorisation de la production laitière en Haute Casamance, Recherches et études sahéliennes $n^{\text {os }} 8$ et 9:149-154.

Dieye P.N., 2006. Arrangements contractuels et performances des marchés du lait local au sud $d u$ Sénégal : les petites entreprises de transformation face aux incertitudes de l'approvisionnement, thèse d'agroéconomie, Montpellier, $211 \mathrm{p}$.

Direction de la Prévision Statistique, 2004. Situation économique et sociale dans la région de Kolda, république du Sénégal, 63p.

Duteurtre G. et al, 2005. « Les bars laitiers de Ndjamena (Tchad), des petites entreprises qui valorisent le lait de brousse ", Raimond C. et al. (dir.) Ressources vivrières et choix alimentaires dans le bassin du lac de Tchad, colloque IRD : 435-455.

Faye A., Fall A., 1992. Les étables fumières en zone de trypanotolérance du Sud du Sénégal. Rapport de recherche, ISRA-CRZ Kolda, 27 p.

Faye B., 2006. Les pasteurs sont des éleveurs « contemplatifs », Courade G. (dir.), L’Afrique des idées reçues, Paris, Belin, 2006 : 281-287.

Fanchette S., 2002. La Haute-Casamance à l'heure de la régionalisation, Diop M. C. (dir.), La société sénégalaise entre le local et le global, Paris, Karthala : 308-355.

Loudiyi S., Angeon V., Lardon S., 2004. « Capital social et développement territorial, quel impact spatial des relations sociales ", communication au colloque le concept de développement en débat, Université Montesquieu-Bordeaux, France, 16-17 septembre. 
Lévy J., Lussault M., 2003. Dictionnaire de la géographie et de l'espace des sociétés, Paris, Belin, 1033 p.

Magrin G., 2007. Rapport de mission à Kolda et à Vélingara, ATP Icare, 31 p.

Vatin F., 1996. Le lait et la raison marchande, Essais de sociologie économique. Rennes, Presses

Universitaires de Rennes, 205 p.

\section{NOTES}

1. Cette étude est financée par une Action Thématique Programmée (ATP) du Centre de coopération internationale en recherche agronomique pour le développement (CIRAD) : ATP Icare (Impact de l'ouverture des marchés sur le développement territorial des régions d'élevage en Afrique occidentale et orientale).

2. Créée en 1974 et privatisée en 2003, la SODEFITEX fait partie de DAGRIS (Développement des Agro-industries du Sud). La SODEFITEX mène toujours des actions de vulgarisation cotonnière et d'encadrement de ses producteurs au Sénégal.

3. Terme que l'on traduit par « développement » en Pulaar.

4. L Etat sénégalais importe près de $70 \%$ de sa consommation de lait sous forme de poudre de lait (Broutin et al, 2005). De 2006 à 2008, le prix du lait en poudre au kilogramme est passé du simple ( 500 FCFA) au double.

5. Les études de terrain ont été effectuées en juillet 2007 et en aout-septembre 2007 dans le cadre d'un master 2 de géographie soutenu en 2008. Actuellement, une thèse de géographie est en cours, portant sur les relations entre commerce des produits animaux et développement régional dans les villes (Kolda et de Dahra) des principales régions d'élevage (Ferlo, Haute Casamance) du Sénégal.

\section{RÉSUMÉS}

Dans la région de Kolda, le commerce du lait de vache s'est progressivement développé grâce à l'existence des ceintures laitières périurbaines et à la création récente d'un " bassin laitier » par une société cotonnière désireuse de diversifier ses activités. Ces espaces de collecte révèlent les stratégies des acteurs du commerce du lait. Ainsi les gérants des mini-laiteries et des centres de collecte du «bassin laitier» mettent-ils en place des processus de collecte et de commercialisation qui varient d'un espace à l'autre, en fonction des moyens financiers et logistiques. Ces stratégies témoignent également de la fragilité des acteurs du commerce laitier.

The trade of milk gradually developed in the Kolda region due to the existence of dairy belts and of a dairy basin around Kolda. The organization and the dynamics of these spaces of milk collection reveal the actors strategies. The mini-dairies's managers and the manifold centers of the dairy basin are developing strategies of collection and marketing which vary from a space to another according to financial means and logistic. hese strategies also reveal the difficulties of the actors. 
INDEX

Mots-clés : bassin laitier, ceinture laitière périurbaine, commerce du lait, mini-laiterie, région de Kolda, Sénégal

Keywords : area of Kolda, dairy basin, dairy belts, mini-dairy, Sénégal, trade of milk

\section{AUTEUR}

\section{NÉNÉ DIA}

Néné Dia (ninidia2003@yahoo.fr) est doctorante en géographie à l'université Université Gaston Berger de Saint-Louis. 